\title{
Acoustic Streaming Induced by Periodic and Aperiodic Sound in a Bubbly Liquid
}

\author{
P. WOJDA* AND A. PERElOMOVA
}

Gdańsk University of Technology, Faculty of Applied Physics and Mathematics

G. Narutowicza 11/12, 80-233 Gdańsk, Poland

(Received December 5, 2013; in final form March 15, 2014)

\begin{abstract}
The vortex flow which follows intense sound propagating in a bubbly liquid, is considered. The reasons for acoustic streaming are both nonlinearity and dispersion. That makes streaming especial as compared with that in a Newtonian fluid. Conclusions concern the vortex flow induced in a half-space by initially harmonic or impulse Gaussian beam. The vortex flow recalls a turbulent flow with increasing in time number of small-scale vortices in the vicinity of the axis of a beam's propagation.
\end{abstract}

DOI: $10.12693 /$ APhysPolA.125.1138

PACS: $43.25 . \mathrm{Yw}$

\section{Introduction}

Acoustic waves in unbounded media may reveal noticeable dispersion under some special conditions [1-3]. That concerns, among other, inhomogeneous media and these with unusual thermodynamic properties, like gases with relaxing oscillatory degrees of a molecule's freedom or fluids with a chemical reaction [4]. A liquid which involves gaseous bubbles is much more compressible than the pure liquid, so that its acoustical properties differ considerably from that in both pure gas or liquid $[2,5]$. The sound speed is fairly smaller than that in a liquid, nonlinearity increases by orders of magnitude, and features of sound propagation strongly depend on its frequency. Sound of finite magnitude is nonlinearly distorted and does interact with the non-wave fluid motions as it propagates. Studies of nonlinear effects are important not only relatively to sound itself, but in connection with phenomena induced in the field of sound.

A model which describes the finite-magnitude sound propagation over a bubbly liquid, imposes inclusion of set of bubbles (i.e., oscillators) into the bulk of a liquid [5-9]. The equation which governs sound beam is analogous to the famous Khokhlov-Zabolotskaya-Kuznetsov (KZK) equation [2, 10, 11], but it includes a dispersive term instead of standard attenuation or supplemented it $[2,10]$. General analytical methods to solve the KZK equation are still absent [12], all the more so solution of equation which governs sound in a bubbly viscous liquid.

Studies of nonlinear fluid dynamics must start from equations describing fluid dynamic of the mixture as a continuum. In three dimensions, both non-wave modes, i.e., vorticity and entropy modes (these names come from the linear theory of flows of Newtonian uniform unbounded fluids [13]) are nonlinearly generated in the field of sound $[14,15]$. The main difficulty is to describe as precisely as possible the nonlinear propagation of sound

*corresponding author; e-mail: pwojda@mif.pg.gda.pl beam itself and interaction of sound and non-wave modes. We derive weakly nonlinear equations for every mode analytically, and use numerical methods to solve them approximately in the context of acoustic streaming. The procedure of subdivision of individual dynamic equations was applied by one of the authors in the problems of acoustic heating and streaming in Newtonian and some non-Newtonian fluids [16-18].

\section{Equations governing perturbations in bubbly liquid}

We consider the mixture which consists of compressible liquid and identical spherical bubbles of an ideal gas. All bubbles are of the same radii at equilibrium, and there is no heat and mass transfer between liquid and gas. We assume also that motions of the bubbles do not influence each other and that they pulsate in their lowest, radially symmetric mode. The mixture as a whole may be treated as the homogeneous continuum. Pressure of the mixture equals pressure of a liquid $[5,19]$. Quantities relating to gas, liquid, or to the mixture, are marked by indices $g$, $l$, and mix, correspondingly. The unperturbed quantities are marked by additional zero, and the disturbed ones are primed. Density of the mixture is given by

$$
\rho_{\text {mix }}=\frac{\rho_{\mathrm{g}} \rho_{\mathrm{l}}}{\beta \rho_{\mathrm{l}}+(1-\beta) \rho_{\mathrm{g}}},
$$

where $\beta$ is the constant mass concentration of gas in the mixture. The initial volume concentration of gas in the mixture, $\alpha$, equals

$$
\alpha=\beta \frac{\rho_{\text {mix } 0}}{\rho_{\mathrm{g} 0}} .
$$

Acoustics of incompressible liquids (when the sound speed in a pure liquid, $c_{1}$, tends to infinity) including bubbles was originally studied by van Wijngaarden [5]. Involving of liquid compressibility corrects the nonlinear parameter of sound $[2,20]$. The following equations in differential form declare conservation of momentum, energy, and mass:

$$
\frac{\partial \boldsymbol{v}}{\partial t}+(\boldsymbol{v} \cdot \boldsymbol{\nabla}) \boldsymbol{v}+\frac{1}{\rho_{\text {mix }}} \nabla p=0
$$




$$
\begin{aligned}
& \frac{\partial p}{\partial t}+c_{1}^{2} \frac{\partial \rho_{\mathrm{l}}}{\partial t}-\frac{c_{\mathrm{l}}^{2}\left(\gamma_{\mathrm{l}}-1\right)}{\rho_{\mathrm{l} 0}} \rho_{\mathrm{l}} \frac{\partial \rho_{\mathrm{l}}}{\partial t}=0, \\
& \frac{\partial \rho_{\text {mix }}}{\partial t}+\nabla\left(\boldsymbol{v} \rho_{\text {mix }}\right)=0,
\end{aligned}
$$

where $\boldsymbol{v}, p$ denote velocity and pressure in the mixture, $\gamma_{1}=\frac{C_{p, 1}}{C_{V, 1}} \frac{\rho_{10}}{p_{10}}\left(\frac{\partial p_{1}}{\partial \rho_{1}}\right)_{T=\text { const }}$, where $C_{p}$ and $C_{V}$ denote heat capacities at constant pressure and density. For water at normal conditions, it equals approximately 7 . Some other equations complement the system (3). The first reflects constant mass of gas inside a spherical bubble, whose density is constantly distributed over a volume ( $R$ denotes a bubble's radius),

$$
R^{3} \rho_{\mathrm{g}}=R_{0}^{3} \rho_{\mathrm{g} 0},
$$

and the second one describes adiabatic behavior of gas inside it,

$$
p_{\mathrm{g}} \rho_{\mathrm{g}}^{-\gamma_{\mathrm{g}}}=p_{\mathrm{g} 0} \rho_{\mathrm{g} 0}^{-\gamma_{\mathrm{g}}},
$$

where $\gamma_{\mathrm{g}}=\frac{C_{p, \mathrm{~g}}}{C_{V, \mathrm{~g}}}$. Equation (5) imposes no energy exchange between bubbles and surrounding liquid. Oscillations of each bubble are described by the RayleighPlesset equation [21]:

$$
\begin{aligned}
& R \frac{\partial^{2} R}{\partial t^{2}}+\frac{3}{2}\left(\frac{\partial R}{\partial t}\right)^{2}-\frac{1}{c_{1}}\left[R^{2} \frac{\partial^{3} R}{\partial t^{3}}+6 R \frac{\partial R}{\partial t} \frac{\partial^{2} R}{\partial t^{2}}\right. \\
& \left.+2\left(\frac{\partial R}{\partial t}\right)^{3}\right]=\frac{p_{\mathrm{g}}^{\prime}-p_{1}^{\prime}}{\rho_{\mathrm{l}}} .
\end{aligned}
$$

Equation (6) accounts for compressibility of a liquid $[6,19]$. Equations $(4)-(6)$ permit to rearrange the second equation from the system (3) in terms of quantities describing the mixture as a whole: $p, \rho_{\text {mix }}, \boldsymbol{v}$. Equations $(3)$ in the dimensionless quantities $(\boldsymbol{x}$ denotes vector of the Cartesian coordinates, $\lambda$ is the characteristic scale of acoustic perturbations, and $c_{\text {mix }}$ is the speed of sound of infinitely small magnitude in a bubbly liquid [5]):

$$
\begin{aligned}
& \boldsymbol{v}^{\mathrm{d}}=\frac{\boldsymbol{v}^{\prime}}{c_{\text {mix }}}, \quad p^{\mathrm{d}}=\frac{p^{\prime}}{c_{\text {mix }}^{2} \rho_{\text {mix } 0}}, \quad \rho^{\mathrm{d}}=\frac{\rho_{\text {mix }}^{\prime}}{\rho_{\text {mix } 0}}, \\
& \boldsymbol{x}^{\mathrm{d}}=\frac{\boldsymbol{x}}{\lambda}, \quad t^{\mathrm{d}}=\frac{t c_{\text {mix }}}{\lambda}, \\
& \frac{1}{c_{\text {mix }}^{2}}=\frac{(1-\alpha)^{2}}{c_{1}^{2}}+\frac{\alpha(1-\alpha) \rho_{\mathrm{l0}}}{\gamma_{\mathrm{g}} p_{\mathrm{g} 0}},
\end{aligned}
$$

take the form [20]:

$$
\begin{aligned}
& \frac{\partial \boldsymbol{v}}{\partial t}+\boldsymbol{\nabla} p=-((\boldsymbol{v} \cdot \boldsymbol{\nabla}) \boldsymbol{v}-\rho \boldsymbol{\nabla} p) \\
& \frac{\partial p}{\partial t}+\boldsymbol{\nabla} \cdot \boldsymbol{v}-\frac{\alpha(1-\alpha) R_{0}^{2} \rho_{\mathrm{l0}}^{2} c_{\mathrm{mix}}^{4}}{3\left(\gamma_{\mathrm{g}} p_{\mathrm{g} 0}\right)^{2}} \frac{\partial^{3} p}{\partial t^{3}} \\
& \quad=(1-\alpha) c_{\text {mix }}^{2}\left(-\frac{\gamma_{\mathrm{l}}+1}{c_{\mathrm{l}}^{2}} \rho \boldsymbol{\nabla} \cdot \boldsymbol{v}-c_{\mathrm{mix}}^{2}\right. \\
& \left.\quad \times \frac{\alpha(1-\alpha) \rho_{\mathrm{l0}}^{2}\left(\gamma_{\mathrm{g}}+1\right)}{\left(\gamma_{\mathrm{g}} p_{\mathrm{g} 0}\right)^{2}} p \boldsymbol{\nabla} \cdot \boldsymbol{v}\right)-\boldsymbol{v} \cdot \boldsymbol{\nabla} \rho+\rho \boldsymbol{\nabla} \cdot \boldsymbol{v}
\end{aligned}
$$

$$
\frac{\partial \rho}{\partial t}+\nabla \cdot(\rho \boldsymbol{v})=0
$$

Starting from Eqs. (9), upper indices by dimensionless quantities will be omitted. The largest, quadratic terms are kept in the non-linear right-hand parts of all equations.

\section{Acoustic and non-wave modes and relative dynamic equations}

For motions of infinitely small magnitudes, the system (9) takes the form

$$
\frac{\partial \Psi}{\partial t}+L \Psi=0
$$

where $\Psi$ is a vector of perturbations, $\Psi=\left(\begin{array}{c}v_{x} \\ v_{y} \\ v_{z} \\ p \\ \rho\end{array}\right)$, and

$$
\begin{aligned}
L & =\left(\begin{array}{ccccc}
0 & 0 & 0 & \frac{\partial}{\partial x} & 0 \\
0 & 0 & 0 & \frac{\partial}{\partial y} & 0 \\
0 & 0 & 0 & \frac{\partial}{\partial z} & 0 \\
\frac{\partial}{\partial x}(1+D \Delta) & \frac{\partial}{\partial y}(1+D \Delta) & \frac{\partial}{\partial z}(1+D \Delta) & 0 & 0 \\
\frac{\partial}{\partial x} & \frac{\partial}{\partial y} & \frac{\partial}{\partial z} & 0 & 0
\end{array}\right), \\
D & =\frac{\alpha(1-\alpha) R_{0}^{2} \rho_{10}^{2} c_{\text {mix }}^{2}}{3\left(\gamma_{\mathrm{g}} p_{\mathrm{g} 0}\right)^{2} \lambda^{2}}
\end{aligned}
$$

are the linear matrix operator including spatial derivatives and the small parameter responsible for dispersion, $D ; \Delta$ denotes the Laplace operator. Studies of motions of infinitely-small amplitudes begin usually with representing of all perturbations as a sum of planar waves

$$
\begin{gathered}
f(\boldsymbol{x}, t)=\int \tilde{f}(\boldsymbol{k}, t) \exp (-\mathrm{i} \boldsymbol{k} \cdot \boldsymbol{x}) \mathrm{d} \boldsymbol{k} \\
=\int \tilde{f}(\boldsymbol{k}) \exp (\mathrm{i} \omega t-\mathrm{i} \boldsymbol{k} \cdot \boldsymbol{x}) \mathrm{d} \boldsymbol{k}
\end{gathered}
$$

$(\tilde{f}(\boldsymbol{k}, t)$ denotes the Fourier transform of $f(\boldsymbol{x}, t)$,

$$
\widetilde{f}(\boldsymbol{k}, t)=\frac{1}{(2 \pi)^{3}} \int f(\boldsymbol{x}, t) \mathrm{e}^{\mathrm{i} \boldsymbol{k} \cdot \boldsymbol{x}} \mathrm{d} \boldsymbol{x},
$$

and $\boldsymbol{k}$ is the wave vector). In all evaluations below only terms proportional to $D^{0}$ and $D^{1}$, are retained. There are five roots of dispersion equation, two first being acoustic (marked by indices 1 and 2, respectively), the third dispersion relation describing stationary (or "entropy") mode, and the last two zero roots describing the stationary vortex motion,

$$
\begin{aligned}
& \omega_{1}=-\mathrm{i} \sqrt{\widetilde{\Delta}}(1+D \widetilde{\Delta} / 2), \quad \omega_{2}=\mathrm{i} \sqrt{\widetilde{\Delta}}(1+D \widetilde{\Delta} / 2), \\
& \omega_{3}=0, \quad \omega_{4}=0, \quad \omega_{5}=0,
\end{aligned}
$$

where

$$
\widetilde{\Delta}=-k_{x}^{2}-k_{y}^{2}-k_{z}^{2}, \quad \sqrt{\widetilde{\Delta}}=\mathrm{i} \sqrt{k_{x}^{2}+k_{y}^{2}+k_{z}^{2}} .
$$

They determine relations of perturbations specific for every mode ( $\widetilde{\Psi}$ denotes a vector of Fourier transforms of perturbations): 


$$
\begin{aligned}
\widetilde{\Psi}_{1}=\left(\begin{array}{c}
\frac{\mathrm{i} k_{x}}{\sqrt{\widetilde{\Delta}}}\left(1+\frac{D}{2} \widetilde{\Delta}\right) \\
\frac{\mathrm{i} k_{y}}{\sqrt{\widetilde{\Delta}}}\left(1+\frac{D}{2} \widetilde{\Delta}\right) \\
\frac{\mathrm{i} k_{z}}{\sqrt{\widetilde{\Delta}}}\left(1+\frac{D}{2} \widetilde{\Delta}\right) \\
1+D \widetilde{\Delta} \\
1
\end{array}\right) \widetilde{\rho}_{1}, \\
\widetilde{\Psi}_{2}=\left(\begin{array}{c}
-\frac{\mathrm{i} k_{x}}{\sqrt{\widetilde{\Delta}}}\left(1+\frac{D}{2} \widetilde{\Delta}\right) \\
-\frac{\mathrm{i} k_{y}}{\sqrt{\widetilde{\Delta}}}\left(1+\frac{D}{2} \widetilde{\Delta}\right) \\
-\frac{\mathrm{i} k_{z}}{\sqrt{\widetilde{\Delta}}}\left(1+\frac{D}{2} \widetilde{\Delta}\right) \\
1+D \widetilde{\Delta} \\
1
\end{array}\right) \widetilde{\rho}_{2}, \widetilde{\Psi}_{3}=\left(\begin{array}{l}
0 \\
0 \\
0 \\
0 \\
1
\end{array}\right) \widetilde{\rho}_{3},
\end{aligned}
$$

where $\widetilde{\rho}_{n}(n=1,2,3)$ are the Fourier transforms of perturbations in density belonging to corresponding specific mode. The both branches of the vortex mode may be determined in the following way (they correspond to the rotational flow of a fluid of constant pressure and density):

$$
\boldsymbol{\nabla} \cdot \boldsymbol{v}_{4,5}=0, \quad p_{4,5}=0, \quad \rho_{4,5}=0,
$$

where $\boldsymbol{v}_{4}, \boldsymbol{v}_{5}$ denote two arbitrary independent branches of the vortex flow: $\boldsymbol{v}_{4}+\boldsymbol{v}_{5}=\boldsymbol{v}_{\text {vort }}$. The both sound modes and the entropy motion are potential. Equations (13), (14) may be expanded in series with respect to powers of $D$. That significantly simplifies evaluations.

The modes of a flow of infinitely small magnitude do not interact far from boundaries. The dynamic equations which govern any mode, may be readily decomposed from the linearized system (10) by use of links specific for each mode. That may be done by means of projectors [20]. The projecting operators point the way for successful decomposing of equations governing every mode also in a weakly nonlinear flow. The projection results to dynamic equations with nonlinear terms responsible for the modes interaction. Equations (9) accounting for nonlinear terms, take the form

$$
\frac{\partial \Psi}{\partial t}+L \Psi=\Psi_{n l}
$$

Application of the "vortex" projector on the system (16) cancels all acoustic and entropy terms in the linear part of the left-side vector, but yields nonlinear source in the right-hand vector. We will consider among nonlinear terms represented by $\Psi_{n l}$ only acoustic ones. That corresponds to intense sound as compared to the non-wave modes. The vorticity projector in fact applies on three components of overall velocity. Its part, applying on the velocity vector, $P_{\mathrm{vort}, \boldsymbol{v}}$, takes the form

$$
P_{\text {vort }, \boldsymbol{v}}=\frac{1}{\Delta}\left(\begin{array}{ccc}
\frac{\partial^{2}}{\partial y^{2}}+\frac{\partial^{2}}{\partial z^{2}} & -\frac{\partial^{2}}{\partial x \partial y} & -\frac{\partial^{2}}{\partial x \partial z} \\
-\frac{\partial^{2}}{\partial x \partial y} & \frac{\partial^{2}}{\partial x^{2}}+\frac{\partial^{2}}{\partial z^{2}} & -\frac{\partial^{2}}{\partial y \partial z} \\
-\frac{\partial^{2}}{\partial x \partial z} & -\frac{\partial^{2}}{\partial y \partial z} & \frac{\partial^{2}}{\partial x^{2}}+\frac{\partial^{2}}{\partial y^{2}}
\end{array}\right) .
$$

Application of $P_{\mathrm{vort}, \boldsymbol{v}}$ on the first three equations from the system (9), which represent the momentum equation, results in the dynamic equation governing velocity of the vorticity mode

$$
\frac{\partial \boldsymbol{v}_{\text {vort }}}{\partial t}=P_{\text {vort }, \boldsymbol{v}}\left(\begin{array}{l}
-(\boldsymbol{v} \cdot \boldsymbol{\nabla}) v_{x}+\rho \frac{\partial p}{\partial x} \\
-(\boldsymbol{v} \cdot \boldsymbol{\nabla}) v_{y}+\rho \frac{\partial p}{\partial y} \\
-(\boldsymbol{v} \cdot \boldsymbol{\nabla}) v_{z}+\rho \frac{\partial p}{\partial z}
\end{array}\right) .
$$

The right-hand side of Eq. (18) includes, in general, terms belonging to both acoustic modes. By use of relation between acoustic pressure and acoustic excess density, it may be rearranged into the following equation:

$$
\frac{\partial \boldsymbol{v}_{\text {vort }}}{\partial t}=D P_{\text {vort }, \boldsymbol{v}}\left(\sum_{n=1}^{2} \rho_{n} \nabla \Delta \sum_{n=1}^{2} \rho_{n}\right) \text {. }
$$

That yields the dynamic equation for the vorticity mode in the field of intense sound, in two equivalent forms,

$$
\begin{aligned}
& \frac{\partial \boldsymbol{\Omega}}{\partial t}=D\left(\nabla \rho_{a}\right) \times\left(\boldsymbol{\nabla} \Delta \rho_{a}\right), \\
& \frac{\partial \boldsymbol{v}_{\text {vort }}}{\partial t}=D P_{\text {vort }, \boldsymbol{v}}\left(\rho_{a} \boldsymbol{\nabla} \Delta \rho_{a}\right),
\end{aligned}
$$

where $\boldsymbol{\Omega}$ is the vorticity of a flow, $\boldsymbol{\Omega}=\boldsymbol{\nabla} \times \boldsymbol{v}_{\text {vort }}$, and $\rho_{a}=\rho_{1}+\rho_{2}$. Application of the last row of $P_{1}[20]$ on Eqs. (9), if only nonlinear terms belonging to the first mode are kept in the nonlinear part, results in the leading-order equation governing an excess density of the first branch of sound

$$
\begin{aligned}
& \frac{\partial \rho_{1}}{\partial t}-\sqrt{\Delta}(1+D \Delta / 2) \rho_{1}+\left[(2 \varepsilon-1) \rho_{1} \nabla \cdot \boldsymbol{v}_{1} / 2\right. \\
& \left.\quad+\boldsymbol{v}_{1} \cdot \nabla \rho_{1} / 2\right]=0
\end{aligned}
$$

where $\varepsilon$ denotes the parameter of nonlinearity,

$$
\begin{aligned}
\varepsilon= & \frac{(1-\alpha) c_{\text {mix }}^{2}\left(\gamma_{1}+1\right)}{2 c_{1}^{2}} \\
& +\frac{c_{\text {mix }}^{4} \alpha(1-\alpha)^{2} \rho_{\mathrm{l0}}^{2}\left(\gamma_{\mathrm{g}}+1\right)}{2\left(\gamma_{\mathrm{g}} p_{\mathrm{g} 0}\right)^{2}} .
\end{aligned}
$$

and square root of Laplacian $(\sqrt{\Delta} f(\boldsymbol{r}, t))$ means integral operator which corresponds to the Fourier transform of $\mathrm{i} \sqrt{k_{x}^{2}+k_{y}^{2}+k_{z}^{2}} \tilde{f}(\boldsymbol{k}, t)$. The parameter of nonlinearity, given by Eq. (22), coincides with that evaluated in [2]. In the study [2], the expression obtained for incompressible liquid is completed by the terms following from the nonlinearity in equations different from the pressure-density relation for the mixture. Unlike, Eq. (22) is immediate result of decomposition of the total system of conservation equations describing compressible liquid including bubbles into specific dynamic equations.

\subsection{Dynamic equations in the quasi-planar geometry of a flow}

Until this point, no restriction concerning a type of flow geometry was done. In the majority of practical applications, a weakly divergent acoustic beam is of interest. Let $y$ designate the nominal axis of the sound beam pointing in the propagation direction, and let $x, z$ be the coordinates perpendicular to that axis. We will assume that all acoustic perturbations vary much faster in the direction of axis $O Y$ than in the direction perpendicular to this axis: $k_{y}^{2} \gg k_{x}^{2}+k_{z}^{2}$. It allows to expand the relations for sound perturbations in the series of powers of the small 
parameter $\mu=\left(k_{x}^{2}+k_{z}^{2}\right) / k_{y}^{2}$, which is responsible for a beam's divergence. Keeping the leading-order terms, one readily rearranges Eq. (21) into the following form:

$$
\frac{\partial \rho_{1}}{\partial t}+\frac{\partial \rho_{1}}{\partial y}+\frac{\Delta_{\perp}}{2} \int \rho_{1} \mathrm{~d} y+\frac{D}{2} \frac{\partial^{3} \rho_{1}}{\partial y^{3}}+\varepsilon \rho_{1} \frac{\partial \rho_{1}}{\partial y}=0,
$$

where $\Delta_{\perp}=\frac{\partial^{2}}{\partial x^{2}}+\frac{\partial^{2}}{\partial z^{2}}$. This equation is in fact one of the form of $\mathrm{KZ}$ (the non-viscous limit of KZK) equation with inclusion of dispersion. The dynamic equation for the vorticity mode in the field of intense sound progressive in the positive direction of axis $O Y$, takes the leading-order form

$$
\frac{\partial \boldsymbol{\Omega}}{\partial t}=D\left(\boldsymbol{\nabla} \rho_{1}\right) \times\left(\boldsymbol{\nabla} \frac{\partial^{2} \rho_{1}}{\partial y^{2}}\right) .
$$

Equation (24) reveals that both nonlinearity and dispersion are necessary conditions of acoustic streaming in a bubbly liquid. The following assumptions will be made regarding the source: it is defined at the plane $y=0$ and is positioned symmetrically with respect to the $y$-axis. The system (Eqs. (23), (24)) may be readily rearranged in the cylindrical geometry with $r=\sqrt{\mu} \sqrt{x^{2}+z^{2}}$ :

$$
\begin{aligned}
& \frac{\partial \rho_{1}}{\partial t}+\frac{\partial \rho_{1}}{\partial y}+\frac{\mu}{2 r} \frac{\partial}{\partial r}\left(r \int \frac{\partial \rho_{1}}{\partial r} \mathrm{~d} y\right)+\frac{D}{2} \frac{\partial^{3} \rho_{1}}{\partial y^{3}} \\
& +\varepsilon \rho_{1} \frac{\partial \rho_{1}}{\partial y}=0 \\
& \frac{\partial \boldsymbol{\Omega}}{\partial t}=\sqrt{\mu} D\left(\begin{array}{c}
\cos \phi \\
0 \\
-\sin \phi
\end{array}\right) F\left(\rho_{1}\right),
\end{aligned}
$$

where

$$
F\left(\rho_{1}\right)=-\frac{\partial \rho_{1}}{\partial r} \frac{\partial^{3} \rho_{1}}{\partial y^{3}}+\frac{\partial \rho_{1}}{\partial y} \frac{\partial^{3} \rho_{1}}{\partial r \partial y^{2}}
$$

and $\phi=\arccos \left(\frac{z}{\sqrt{x^{2}+z^{2}}}\right)$. The main difficulty in solution for $\Omega$ is establishment of function $\rho_{1}$, which satisfies Eq. (25) and has not analytical solutions. We rearrange Eq. (25) into the following equivalent form:

$$
\begin{aligned}
& \frac{\partial \rho_{1}}{\partial t}+\frac{\partial \rho_{1}}{\partial y}+\frac{\sqrt{\mu}}{2 r} \frac{\partial}{\partial r}(r g)+\frac{D}{2} \frac{\partial^{3} \rho_{1}}{\partial y^{3}}+\varepsilon \rho_{1} \frac{\partial \rho_{1}}{\partial y}=0 \\
& \frac{\partial g}{\partial y}=\sqrt{\mu} \frac{\partial \rho_{1}}{\partial r}
\end{aligned}
$$

where

$$
g=-\sqrt{\mu} \int_{y}^{\infty} \frac{\partial \rho_{1}}{\partial r} \mathrm{~d} y
$$

After establishing of the solution $\rho_{a}$, one can find vorticity solving equations

$$
\frac{\partial \Omega_{y}}{\partial t}=0, \quad \frac{\partial \Omega_{r}}{\partial t}=0, \quad \frac{\partial \Omega_{\phi}}{\partial t}=\sqrt{\mu} D F\left(\rho_{a}\right),
$$

where $\Omega_{y}, \Omega_{r}$, and $\Omega_{\phi}$ are $y$-component, radial component and angular components of vorticity, correspondingly. In order to establish the vortex motion, one has to solve the system of Poisson equations. In the Cartesian coordinates, that requires

$$
\Delta \boldsymbol{v}_{\mathrm{vort}}=-\nabla \times \boldsymbol{\Omega} \text {. }
$$

If $\boldsymbol{\Omega}(t=0, y, r)=\mathbf{0}, \boldsymbol{\Omega}$ can be expressed in terms of angular component of vorticity

$$
\boldsymbol{\Omega}=\left(\begin{array}{c}
\cos \phi \\
0 \\
-\sin \phi
\end{array}\right) \Omega_{\phi} .
$$

The vortex velocity satisfies the following set of equations in the cylindrical coordinates:

$$
\begin{aligned}
& \frac{\partial^{2} v_{y, \text { vort }}}{\partial y^{2}}+\mu \frac{1}{r} \frac{\partial}{\partial r}\left(r \frac{\partial v_{y, \text { vort }}}{\partial r}\right)=-\frac{\sqrt{\mu}}{r} \frac{\partial}{\partial r}\left(r \Omega_{\phi}\right), \\
& \frac{\partial^{2} v_{r, \text { vort }}}{\partial y^{2}}+\mu \frac{\partial}{\partial r}\left(\frac{1}{r} \frac{\partial}{\partial r}\left(r v_{r, \text { vort }}\right)\right)=\frac{\partial \Omega_{\phi}}{\partial y} .
\end{aligned}
$$

The angular component of vortex velocity equals zero.

\section{Numerical evaluations}

We consider initially unperturbed liquid containing gas bubbles. All calculations relate to a circular acoustic transducer at which an excess acoustic density takes the shape of a Gaussian beam. Solutions are obtained in the case of the following set of initial and boundary conditions:

$$
\begin{aligned}
& \rho_{a}(t=0, y, r)=0, \quad \boldsymbol{\Omega}(t=0, y, r)=\mathbf{0}, \\
& \rho_{a}(t, y=0, r)=M \sin (2 \pi t) \mathrm{e}^{-r^{2}-\delta t^{2}}, \\
& \frac{\partial \rho_{a}}{\partial r}(t, y, r=0)=0, \\
& \Omega_{\phi}(t, y=0, r)=0, \quad \Omega_{\phi}(t, y, r=0)=0, \\
& v_{y, \text { vort }}(t, y=0, r)=0, \quad \frac{\partial v_{y, \text { vort }}}{\partial r}(t, y, r=0)=0, \\
& v_{r, \text { vort }}(t, y, r=0)=0 .
\end{aligned}
$$

The parameter $\delta$ is responsible for the deviation of a beam from strictly periodic: $\delta=0$ corresponds to a harmonic at a transducer sound beam. In order to obtain boundary condition for radial component of velocity at the axis of a beam, $v_{r, \text { vort }}(t, y=0, r)$, one should establish a solution of Eq. (33) and substitute it into the following equality:

$$
\frac{\partial v_{r, \text { vort }}}{\partial y}-\sqrt{\mu} \frac{\partial v_{y, \text { vort }}}{\partial r}=\Omega_{\phi}
$$

which in fact is the definition of the angular component of vorticity. Solutions for excess acoustic density and vorticity, Eqs. (28), (29), (31), have been obtained by means of a numerical scheme which uses an implicit Runge-Kutta method, and the second-order central difference scheme in evaluations of spatial derivatives. The method was adapted specially for a flow of a bubbly liquid. To establish a solution of Eqs. (33), (34), the second-order central difference scheme was used in evaluations of spatial derivatives along with the bi-conjugate gradient method (Bi-CG) which has been described in [22].

$\mathrm{Bi}-\mathrm{CG}$ is one of the Krylov subspace methods which makes it possible to obtain solutions for matrix equation $A x=f$ with asymmetric matrix operator $A$. In the context of the problem, equation $A x=f$ repre- 
sents the discrete Poisson equation in cylindrical coordinates. $A$ denotes the discrete version of the Laplace operator (in cylindrical coordinates), which is different in Eqs. (33), (34). Dimension of $A$ equals to number of nodes, $N$, which are used in calculations, $x$ consists of discrete data vector of the longitudinal components (in Eq. (33)) or the radial components of the vortex velocity (in Eq. (34)), and $f$ denotes the discrete form of the right side of Eq. (33) and Eq. (34), respectively.

We start from establishing initial values of $x\left(x_{0}\right)$ and two other vectors, $\tilde{x}_{0}$ and $\tilde{f}$. Further, residues $r_{0}$ and $\tilde{r}_{0}$ and vectors $p_{0}, \tilde{p}_{0}, p_{k}, \tilde{p}_{k}$ are established, which are compounds of vectors $x$ and $\tilde{x}_{0}$ :

$$
\begin{aligned}
& r_{0}=f-A x_{0}, \quad \tilde{r}_{0}=\tilde{f}-A^{\mathrm{T}} \tilde{x}_{0}, \\
& p_{0}=r_{0}, \quad \tilde{p}_{0}=\tilde{r}_{0} .
\end{aligned}
$$

The following actions are repeated until convergence of the vector $x_{k}$ to the solution $x$ ( $k$ is the natural number):

$$
\begin{aligned}
& \alpha_{k}=\frac{\tilde{r}_{k}^{\mathrm{T}} r_{k}}{\tilde{p}_{k}^{\mathrm{T}} A p_{k}}, \\
& x_{k+1}=x_{k}+\alpha_{k} p_{k}, \quad r_{k+1}=r_{k}-\alpha_{k} A p_{k}, \\
& \tilde{r}_{k+1}=\tilde{r}_{k}-\alpha_{k} A^{\mathrm{T}} \tilde{p}_{k}, \quad \beta_{k}=\frac{\tilde{r}_{k+1}^{\mathrm{T}} r_{k+1}}{\tilde{r}_{k}^{\mathrm{T}} r_{k}}, \\
& p_{k+1}=r_{k+1}-\beta_{k} p_{k}, \quad \tilde{p}_{k+1}=\tilde{r}_{k+1}-\beta_{k} \tilde{p}_{k} .
\end{aligned}
$$

For discrete Poisson equation, the counter of repetition $k$ is proportional to the square root of amount of nodes, $N$. That means that the procedure of solution requires $\mathrm{O}\left(N^{3 / 2}\right)$ operations. Calculations are finished when the norm of residuum $r_{k}$ gets smaller than $10^{-10}$. In fact we arrive at solution of discrete Poisson equation in cylindrical coordinates with mixed boundary conditions: the Dirichlet boundary conditions and the Neumann boundary conditions for longitudinal and radial components of the vortex velocity. Solutions of this kind of problem are not available in any commercial software which has been developed for the Poisson equation.

In accordance to the system (26), the angular component of vorticity is proportional to $M^{2}, D$, and $\sqrt{\mu}$. It can be concluded from numerical simulations that vorticity achieves maximum at some distance from a transducer and the axis of a beam. Vorticity decreases far from transducer because of decrease in the magnitude of the acoustic density at large distances due to diffraction. The diffraction parameter $\mu$ also influences on the formation of vorticity. The smaller value of this parameter is, the sound beam is more concentrated near the axis $O Y$, and it generates maximal vorticity at larger distances from a transducer. Oscillations of the vorticity and the vortex velocity generated by pulses are much smaller than that caused by harmonic at transducer acoustic waves.

For periodic acoustic waves, vorticity constantly increases in time at any point. It tends to some limit level in the case of aperiodic sound. That reflects the decrease with time of magnitude of impulse sound.

The numerical results were obtained for water with bubbles; they relate to the following values of parame-

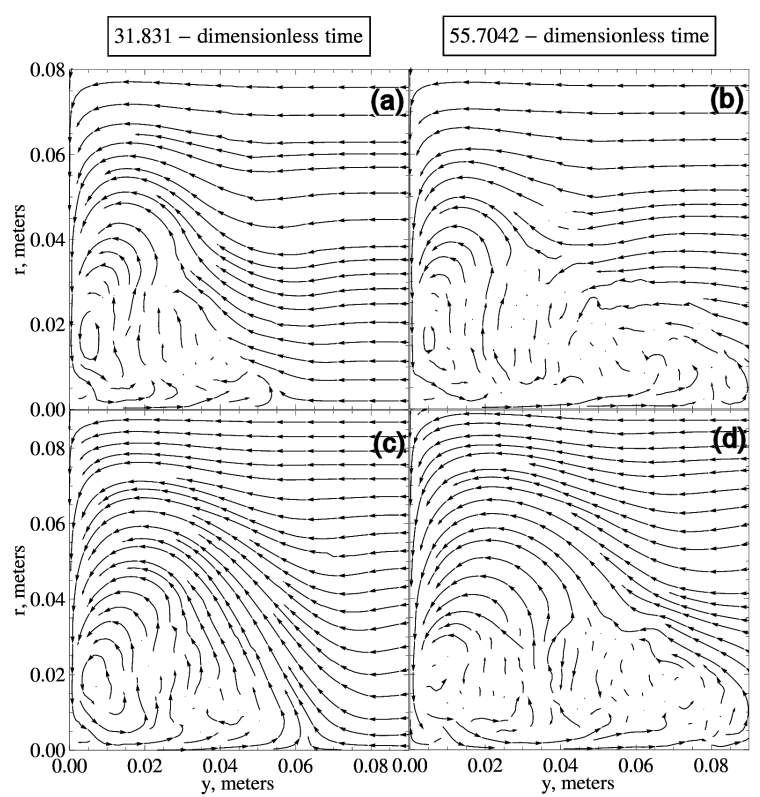

Fig. 1. Streamlines for $\mu=0.0025, \delta=0$ (a,b) and $\delta=0.001(\mathrm{c}, \mathrm{d})$.

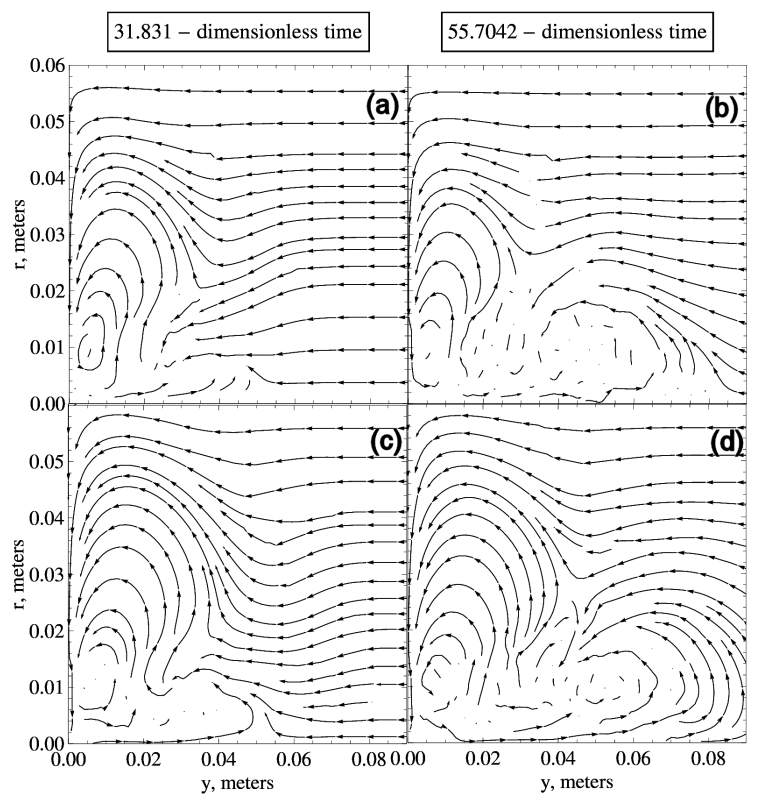

Fig. 2. Streamlines for $\mu=0.01, \delta=0(\mathrm{a}, \mathrm{b})$ and $\delta=$ $0.001(\mathrm{c}, \mathrm{d})$.

ters: $\alpha=0.001, R=2.5 \times 10^{-4} \mathrm{~m}, \rho_{\mathrm{l}}=1000 \mathrm{~kg} / \mathrm{m}^{3}$, $\gamma_{\mathrm{g}}=1.4, p_{\mathrm{g}}=10^{5} \mathrm{~Pa}, c_{\mathrm{l}}=1500 \mathrm{~m} / \mathrm{s}, \lambda=0.00125 \mathrm{~m}$. The sound speed in a gaseous water $c_{\text {mix }}$ equals $374 \mathrm{~m} / \mathrm{s}$ (this means that the frequency of acoustic wave is about $300 \mathrm{kHz}$ ), the dispersion parameter is $D=0.000095$, and the nonlinearity parameter $\varepsilon$ equals 1200 .

Figures 1 and 2 show streamlines of the vortex motion generated by periodic sound and impulses for values of diffraction parameter $\mu$ equal to 0.01 and 0.0025 , respectively ( $\delta$ equals 0 or 0.001 ). Calculation were made 
for the Mach number $M$ which equals 0.0005. Acoustic streaming in the both cases, caused by periodic or aperiodic sound, reveals some general features. Along with the turning of stream towards a transducer far from the axis of a beam, there appear small-scale vortices close to the axis. The direction of streamlines in these small vortices is counterclockwise in the upper vertical cross-section of a flow. The pictures representing impulse sound, are more prolongated in the vertical direction. Streamlines at different times differ by the number of small-scale vortices in the vicinity of the axis. Their number grows with time, and the flow near the axis of a beam recalls a turbulent flow.

\section{Conclusions}

In this study, we consider acoustic streaming caused by periodic and impulse sound in a compressible liquid including gaseous bubbles. Equations (20), which describe nonlinear generation of the vortex mode in the field of intense sound, are instantaneous, and their derivation does not require averaging over the sound period. Neither viscosity of pure phases, nor heat transfer between bubbles and surrounding liquid, nor non-uniformity of pressure and temperature inside a bubble, nor vaporization in the case of bubbles including vapor, were considered. The nonlinear generation of the vortex mode is caused exclusively by dispersion. In some sense, dispersion acts like a Newtonian attenuation: it is a necessary (along with nonlinearity) condition for generation of the vortex motion in the field of sound. In contrast to a Newtonian attenuation, pure dispersion influences only on the phase speed of different harmonics of sound, but not on their attenuation. Both nonlinearity and dispersion participate in a nonlinear transfer of momentum and energy between sound and non-wave modes, but this transfer is not so effective as in Newtonian fluids, because it does not impose irreversible losses of momentum and energy of a flow as a whole. This is a reason for the acoustic streaming to reveal features of the turbulent flow: formation of a series of increasing number of small-scale vortices takes place. That happens also to flows of a Newtonian fluid, when nonlinearity is large and attenuation does not longer stabilize a flow, i.e., in flows with high Reynolds numbers. Dispersion in its pure form represents somewhat artificial model: in general, dispersion and attenuation in acoustics flow are connected, like they are not independent in optics [10]. We do not consider convective nonlinearity in the left-hand side of dynamic equation of vorticity, Eq. (24), which is described by the term $(\boldsymbol{v} \cdot \boldsymbol{\nabla}) \boldsymbol{\Omega}$. It is well established that account for this term prevents enlargement of vorticity with time [23].

Numerical results show impact of non-linearity, dispersion and diffraction of acoustic beam on the vortex motion. The results concern the free half-space with circular transducer which is situated at the boundary $y=0$. Inclusion of boundaries may essentially change conclusions and influences on the very definition of modes which as a rule refers to a discrete set of wave numbers in depen- dence of the geometry of a volume and boundary conditions. Preliminary analysis of results has revealed impact of the ratio of the Mach number and dispersion parameter on the vorticity mode. If the Mach number of a flow $M$ is larger than dispersion $D$, streamlines may change direction in some domains. Another remarkable conclusion concerns influence of diffraction. The smaller value of the diffraction parameter is, vorticity achieves maximum at larger distance from a transducer, and its variations are noticeable at the smaller distances from the axis $O Y$.

\section{References}

[1] L.I. Mandelshtam, M.A. Leontowich, JETP 7, 438 (1937).

[2] M. Hamilton, Y. Ilinskii, E. Zabolotskaya, in: Nonlinear Acoustics, Eds. M. Hamilton, D. Blackstock, Academic Press, New York 1998, p. 151.

[3] S.B. Leble, Nonlinear Waves in Waveguides: with Stratification, Springer-Verlag, Berlin 1991.

[4] A.I. Osipov, A.V. Uvarov, Sov. Phys. Usp. 35, 903 (1992).

[5] L. van Wijngaarden, Acta Appl. Math. 39, 507 (1995).

[6] J.B. Keller, M. Miksis, J. Acoust. Soc. Am. 68, 628 (1980).

[7] R.I. Nigmatulin, N.S. Khabeev, F.B. Nagiev, Heat Mass Transf. 24, 1033 (1981).

[8] M. Plesset, A. Prosperetti, Ann. Rev. Fluid Mech. 9, 145 (1977).

[9] E.A. Zabolotskaya, S.I. Soluyan, Sov. Phys. Acoust. 18, 396 (1973)

[10] O.V. Rudenko, S.I. Soluyan, Theoretical Foundations of Nonlinear Acoustics, Plenum, New York 1977.

[11] V.P. Kuznetsov, Sov. Phys. Acoust. 16, 467 (1971).

[12] M. Hamilton, V. Khokhlova, O.V. Rudenko, J. Acoust. Soc. Am. 101, 1298 (1997).

[13] B.-T. Chu, L.S.G. Kovasznay, J. Fluid. Mech. 3, 494 (1958).

[14] P. Marmottant, J.P. Raven, H. Gardeniers, J.G. Bomer, S. Hilgenfeldt, J. Fluid Mech. 568, 109 (2006).

[15] D. Ahmed, X. Mao, J. Shi, B.K. Juluria, T.J. Huang, Lab Chip 9, 2738 (2009).

[16] A. Perelomova, Acta Acustica united with Acustica 89, 754 (2003).

[17] A. Perelomova, Acta Acustica united with Acustica 96, 43 (2010).

[18] A. Perelomova, Canad. J. Phys. 88, 293 (2010).

[19] A. Prosperetti, A. Lezzi, J. Fluid Mech. 168, 457 (1986).

[20] A. Perelomova, Appl. Math. Lett. 13, 93 (2000).

[21] T.G. Leighton, The Acoustic Bubble, Academic Press, New York 1994

[22] R. Fletcher, "Conjugate gradient methods for indefinite systems", Lect. Notes Math. 506, 73 (1976).

[23] T. Kamakura, K. Matsuda, Y. Kumamoto, M.A. Breazeale, J. Acoust. Soc. Am. 97, 2740 (1995). 